\title{
Entanglement susceptibility: Area laws and beyond
}

\section{Paolo Zanardi and Lorenzo Campos Venuti}

Department of Physics and Astronomy, and Center for Quantum Information Science \& Technology, University of Southern California, Los Angeles, CA 90089-0484

\begin{abstract}
Generic quantum states in the Hilbert space of a many body system are nearly maximally entangled whereas low energy physical states are not; the so-called area laws for quantum entanglement are widespread. In this paper we introduce the novel concept of entanglement susceptibility by expanding the 2-Renyi entropy in the boundary couplings. We show how this concept leads to the emergence of area laws for bi-partite quantum entanglement in systems ruled by local gapped Hamiltonians. Entanglement susceptibility also captures quantitatively which violations one should expect when the system becomes gapless. We also discuss an exact series expansion of the 2-Renyi entanglement entropy in terms of connected correlation functions of a boundary term. This is obtained by identifying Renyi entropy with ground state fidelity in a doubled and twisted theory.
\end{abstract}




\section{Introduction}

The quantum-mechanical state space of a many-body system is huge, but an exponentially large fraction of it is inhabited by fairly unphysical states [1]. Roughly speaking one may say that, since we live in a relatively cold universe ruled by local quantum field theory, the vast majority of states one encounters in physically relevant situations are highly non generic as they are low-energy states of local Hamiltonians.

For example it has been known for some time now that generic random quantum states are nearly maximally entangled 2 and that this generic property implies a so-called volume law for the entanglement entropy $S$. More specifically partitioning a system into two regions $A$ and $B$ with $N_{A}$ and $N_{B}$ spins respectively and a with a common boundary $\partial A$, the entanglement between $A$ and $B$ is $O(\min \{|A|,|B|\})$. At variance with this generic prediction, area laws i.e., $S=O(|\partial A|)$, for quantum entanglement (as well as other information theoretic quantities [3]) are ubiquitous in physics: from black hole thermodynamics to quantum entanglement in zerotemperature many body systems (see [4] for a review).

Moreover also violations of these laws have a fundamental physical meaning, as they signal the divergence of an underlying correlation length e.g., quantum criticality [5] or give rise to sub-leading terms that allow one to detect hidden topological order [6]. Ensembles of physical random states have recently been shown to feature an area law in the typical case [7]. Finally, the very fact that area laws hold for many systems of interest i.e., the relevant states are just slightly entangled, is the key feature at the grounds of efficient classical simulations algorithms for quantum systems 8 .

While locality and finite range correlations for gapped system certainly provide an intuitive ground for understanding the origin of area laws, proving them in a rigorous fashion is often quite hard [9]. For the sake of concreteness let us focus on the ground state of a local Hamiltonian: what makes it different from a generic quantum states? More specifically, why is an area law for entanglement obeyed as opposed to a volume law?

The goal of this paper is to show how one can produce a surprisingly simple argument for the emergence of bi-partite entanglement area laws for low-energy states e.g., ground states of gapped systems with local Hamiltonians. The argument we are going to discuss is both quantitative and general and is based on elementary perturbative expansion of the 2-Renyi entropy with respect the boundary Hamiltonian terms. This allows one to introduce a natural notion of entanglement susceptibility that in turn, and in spite of its simplicity, is able to unveil scaling behavior. Remarkably entanglement susceptibility also shows in which ways area laws might be violated for gapless systems and excited energy eigenstates.

In this paper we also discusses an intimate connection between bi-partite entanglement and ground state fidelity [10] as well as a relation between entanglement susceptibility and fidelity susceptibility [11, 12. Fidelity and entanglement are two of the most fundamental quantum information tools and both can be exploited to study quantum phase transitions [13. Entanglement shows that at criticality unique quantum correlations become long-ranged and fidelity witnesses the ensuing orthogonality catastrophe in the Hilbert space. We find it conceptually rewarding that these two phenomena appear now as two sides of the same coin. 


\section{Entanglement susceptibility}

Let us consider a spin system with state-space $\mathcal{H}_{\Lambda}:=\otimes_{i \in \Lambda} h_{i} \cong\left(\mathbf{C}^{d}\right)^{\otimes|\Lambda|}$ and let $(A, B)$ be a bi-partition of the set of vertices $\Lambda$ such that, with obvious notation, $\mathcal{H}_{\Lambda}=\mathcal{H}_{A} \otimes \mathcal{H}_{B}$. Any local Hamiltonian $H=\sum_{X \subset \Lambda} H_{X}$ over $\mathcal{H}_{\Lambda}\left(H_{X} \in B\left(\mathcal{H}_{X}\right)\right.$ can be written as $H=H_{A}+H_{B}+H_{\partial}$ where $H_{A / B}=\sum_{X \subset A / B} H_{X}$ and $H_{\partial}=$ $\sum_{X \cap A \cap B \neq \emptyset} H_{X}$. We are now interested in the eigen-state properties of the following family of Hamiltonians associated to $H$ :

$$
H(\lambda)=H_{A}+H_{B}+\lambda H_{\partial}, \quad(\lambda \in[0,1])
$$

Of course $H(0):=H_{0}$ describes the situation in which the two regions $A$ and $B$ are decoupled whereas $H(1)$ is nothing but the original Hamiltonian where boundary coupling $H_{\partial}$ is assumed to be non-trivial. The eigenstates of $H_{0}$ have the factorized form $\left|p_{A}\right\rangle\left|p_{B}\right\rangle$ with eigenvalues $E_{p_{A}}+E_{p_{B}}$, the non-degenerate ground state being $\left|\Psi_{0}\right\rangle=\left|0_{A}\right\rangle\left|0_{B}\right\rangle$ with energy $E_{0}$. Let $\left|\Psi_{0}(\lambda)\right\rangle$ denote the ground state of (1) and $\rho_{A}:=$ $\operatorname{Tr}_{B}\left|\Psi_{0}(\lambda)\right\rangle\left\langle\Psi_{0}(\lambda)\right|$ the reduced density matrix of the region $A$. In order to quantify the entanglement between $A$ and $B$ we use the Renyi 2-entropy $S_{2}=-\log \operatorname{Tr} \rho_{A}^{2}$. The first result we would like to present is:

$$
\begin{aligned}
& S_{2}(\lambda)=2 \lambda^{2} \chi_{E}+O\left(\lambda^{3}\right) \\
& \chi_{E}:=\sum_{p_{A}, p_{B} \geq 1}\left|\frac{\left\langle p_{A} p_{B}\left|H_{\partial}\right| 0_{A} 0_{B}\right\rangle}{E_{p_{A}}+E_{p_{B}}-E_{0}}\right|^{2}
\end{aligned}
$$

In the remainder of the paper we will refer to this quantity as entanglement susceptibility [14. The derivation of (2) is straightforward based on RayleighSchrödinger perturbation theory and is given in the Appendix. Now a couple of simple qualitative comments:

1) The entanglement susceptibility (2) depends explicitly on the Hamiltonian term $H_{\partial}$. This dependence makes manifest the intuitive fact that entanglement between $A$ and $B$ is caused by interactions terms supported on the boundary between them.

2) The structure of (2) clearly indicates that large gaps (density of states) above the ground state energy may result in small (large) value for $\chi_{E}$. It should also be clear, that vanishing gaps may induce singular behavior of $\chi_{E}$ depending on the behavior of the corresponding matrix elements of $H_{\partial}$. This last fact establishes a direct and transparent connection between the behavior of entanglement susceptibility and quantum criticality.

\section{The area law bound}

To obtain the area law from (2) we introduce the so-called fidelity susceptibility [11, 15] $\chi_{F}:=-\left.\partial^{2} \log \mathcal{F}(\lambda) \partial \lambda^{2}\right|_{\lambda=0}$, where $\mathcal{F}(\lambda):=\left|\left\langle\Psi_{0}(\lambda) \mid \Psi_{0}\right\rangle\right|$ is the ground state fidelity. $\mathcal{F}$ is the main ingredient of the so called fidelity approach to quantum critical phenomena [10] and has been thoroughly investigated over the last few years. The basic fact is that:

$$
\chi_{E} \leq \chi_{F}
$$

Indeed $\chi_{F}$, for the Hamiltonian family (1) at $\lambda=0$, is given by $\sum_{p_{A}+p_{B} \geq 1}\left|\frac{\left\langle p_{A} p_{B}\left|H_{\partial}\right| 0_{A} 0_{B}\right\rangle}{E_{p_{A}}+E_{p_{B}}-E_{0}}\right|^{2}$ [11] that has the very same structure of the term we are willing to bound but without the restriction that both $p_{A}$ and $p_{B}$ are larger than zero 
[16]. The bound (3) is not always tight. In fact, in order to have potential singularities in $\chi_{E}$ the gaps of subsystem $A$ and $B$ have to vanish. On the contrary for $\chi_{F}$ the closure of the gap in any of the two subsystem e.g., say the largest one is approaching criticality, may be enough to generate a divergent behavior. Now we show how the bounds proven in [12] can be adapted to the current case to provide an area law. One can write $\chi_{F}=\| G_{0} H_{\partial}\left|\Psi_{0}\right\rangle \|^{2}$ where $G_{0}=\sum_{p>0}|p\rangle\langle p| /\left(E_{p}-E_{0}\right)$, and observe that for gapped systems $\Delta:=E_{1}-E_{0}>0$ and $\left\|G_{0}\right\|:=\sup _{\|\psi\|=1}\left\|G_{0} \psi\right\| \leq \Delta^{-1}$ from which one has

$$
\begin{aligned}
\chi_{F} & \leq\left\|G_{0}\right\|^{2} \| Q H_{\partial}\left|\Psi_{0}\right\rangle \|^{2} \leq \Delta^{-2}\left\langle\Psi_{0}\left|H_{\partial} Q H_{\partial}\right| \Psi_{0}\right\rangle \\
& =\Delta^{-2}\left(\left\langle\Psi_{0}\left|H_{\partial}^{2}\right| \Psi_{0}\right\rangle-\left\langle\Psi_{0}\left|H_{\partial}\right| \Psi_{0}\right\rangle^{2}\right) .
\end{aligned}
$$

This last factor is nothing but the connected correlation function for the boundary operator $H_{\partial}$ in the ground state $\Psi_{0}$. Thanks to the exponential decay of correlations for gapped ground states of local Hamiltonians [17 the connected correlation above scales as the support of the involved operators. More precisely if $H_{\partial}=\sum_{j \in \partial A} h_{j}$ then $\left|\left\langle H_{\partial} H_{\partial}\right\rangle_{c}\right| \leq \xi^{d-1} \max _{j \in \partial A}\left\|h_{j}\right\|^{2}|\partial A|$, where $\xi$ is of the order of the finite correlation length of the system measured in units of some microscopic length scale e.g., lattice spacing. Therefore, using (3), one gets

$$
\chi_{E} \leq \frac{1}{\Delta^{2}} \max _{j \in \partial A}\left\|h_{j}\right\|^{2} \xi^{d-1}|\partial A|,
$$

In words: the entanglement susceptibility for a gapped (non degenerate) ground state of a local Hamiltonian obeys an area law. We would like to emphasize that this argument shows that violations of the area law i.e., of the bound (5), are a sufficient condition for criticality (for local Hamiltonians). On the other in 12] we showed that in the case in which $H_{\partial}$ has a large scaling dimension criticality does not necessarily imply super-extensive scaling of fidelity susceptibility. In the present case this suggests that one may have gapless systems that still obey an area law. Such phenomena have already been pointed out in the context of projected entangled pair states in [18] or for topological insulators which have gapless edge modes but a finite correlation length in the bulk [19].

Another interesting point is the validity of these results for excited Hamiltonian eigenstates. The derivation of (2) shows that as long as the excited state is energetically well separated from the rest of the spectrum (2) holds true. Moreover if the eigenstate is also clustering i.e., connected correlations functions of local operators decays sufficiently fast, also the derivation of (5) is valid and hence an area law fulfilled. The way this may fail is a high (unperturbed) density of states that makes the excited state (quasi-)degenerate and/or a lack of clustering. These remarks are consistent with the findings of [20].

\section{Example: Quasi-free systems}

The area law prediction of Eq (5) for gapped local Hamiltonian can be confirmed by explicit, and independent, calculations for exactly solvable models. In this section, for the sake of illustration, we discuss two cases:

a) Free fermions on a graph $\Lambda$ with quadratic Hamiltonian $H=\sum_{i, j \in \Lambda} Z_{i j} c_{i}^{\dagger} c_{j}$.

The $c_{i}$ 's are canonical fermionic operators i.e. $\left\{c_{i}, c_{j}^{\dagger}\right\}=\delta_{i j},\left\{c_{i}, c_{j}\right\}=0$ and $Z=\left(Z_{i, j}\right)_{i, j}$ is a real symmetric $|\Lambda| \times|\Lambda|$ matrix, local on $\Lambda$ [27. The analog of equation (11) in this case is given by $Z(\lambda)=Z+\lambda \delta Z$ where $\delta Z$ is with non vanishing 
entries on a $O(|\partial A| \times|\partial A|)$ sub-block $(\operatorname{rank}(\delta Z)=O(|\partial A|)$. The gapfulness assumption in this single particle picture amounts to the invertibility of $Z$ i.e., the minimum $Z$ singular value $\Delta$ is bounded away from zero. The fidelity susceptibility is known to have the form $\chi_{F}=\|\partial T / \partial \lambda\|_{2}^{2}$ where $T(\lambda):=Z|Z|^{-1}$ is the unitary part of the polar decomposition of $Z$ and $\|X\|_{2}:=\sqrt{\operatorname{Tr} X^{\dagger} X}$ [29]. We can now use the bound (see chapter VII.5 of [28])

$$
\left\|T_{A}-T_{B}\right\|_{2} \leq \frac{2}{\left\|A^{-1}\right\|^{-1}+\left\|B^{-1}\right\|^{-1}}\|A-B\|_{2}
$$

(with $\|X\|$ indicating the operator norm of $X$ ), for $A, B$ invertible operators with unitary factors $T_{A, B}$ in their polar decomposition. Setting $A=Z$ and $B=Z+\delta Z$ in the above inequality we get $\|\delta T\|_{2} \leq 2 /\left(\Delta+\Delta^{\prime}\right)\|\delta Z\|_{2}$ with $\Delta^{\prime}$ indicating the smallest singular value of $Z+\delta Z$. Moreover $\|\delta Z\|_{2}^{2} \leq \operatorname{rank}(\delta Z)\|\delta Z\|^{2}$ with $\|\delta Z\|=O(1)$. Thanks to (3) this allows one to conclude

$$
\chi_{E} \leq \frac{4}{\left(\Delta+\Delta^{\prime}\right)^{2}} \operatorname{rank}(\delta Z)\|\delta Z\|^{2}=\frac{1}{\Delta^{2}} O(|\partial A|) .
$$

b) Quasi-free bosons. In this case the Hamiltonian is given by $H=1 / 2 \sum_{i \in \Lambda} p_{i}^{2}+$ $1 / 2 \sum_{i, j \in \Lambda} V_{i j} x_{i} x_{j},\left[x_{i}, p_{j}\right]=i \hbar \delta_{i j}$. The harmonic coupling matrix $V$ is symmetric, non negative definite, local on $\Lambda$ and $\|V\|=O(1)$. The ground state is known to be a Gaussian state $|V\rangle$ with covariance matrix $\Gamma(V)=V^{-1 / 2} \oplus V^{1 / 2}$ and the gap with the first excited state is given by $\Delta=2 \lambda_{\min }\left(V^{1 / 2}\right)\left(\lambda_{\min }(X):=\right.$ minimum eigenvalue of $X$ ) [30. The ground state fidelity can be obtained by performing a simple Gaussian integral and yields: $\mathcal{F}=\left|\left\langle V \mid V^{\prime}\right\rangle\right| \sim\left[\operatorname{det}\left(\Gamma^{-1}+\left(\Gamma^{\prime}\right)^{-1}\right)\right]^{-1 / 4}$; setting $\Gamma^{\prime}:=\Gamma(V+\delta V)=\Gamma+\delta \Gamma$ and expanding at leading order in $\delta \Gamma$ one finds for the fidelity susceptibility: $\chi_{F} \sim\left\|\Gamma^{-1} \delta \Gamma\right\|_{2}^{2}$. This quantity can be bounded above as follows: $\chi_{F} \leq 1 / \lambda_{\min }^{2}(\Gamma)\|\delta \Gamma\|_{2}^{2}=O\left(\|\delta \Gamma\|_{2}^{2} / \Delta^{2}\right)$. Moreover, from the expression of $\Gamma(V)$ above, one obtains $\delta \Gamma=1 / 2\left(V^{-1 / 2} \delta V \oplus V^{-3 / 2} \delta V\right)$ whence $\|\delta \Gamma\|_{2}=1 / 2\left(\left\|V^{-1 / 2} \delta V\right\|_{2}+\left\|V^{-3 / 2} \delta V\right\|_{2}\right)=O\left(\Delta^{-3 / 2}\|\delta V\|_{2}\right)$. Finally, $\chi_{E} \leq \chi_{F}=$ $O\left(\Delta^{-5} \operatorname{rank}(\delta V)\|\delta V\|^{2}\right)=O(|\partial A|)$. In the last steps we exploited Eq. (3) and the fact that the boundary perturbation $\delta V$ has rank $O(|\partial A|)$ and $O(1)$ norm.

\section{Area law corrections in gapless systems}

In this section we would like to illustrate how the entanglement susceptibility (2) is able to capture the modifications to the strict area law that one expects in critical i.e., gapless systems. To this aim let us consider a $d$-dimensional tight-binding model on a hypercubic lattice. We partition the system into two half-spaces $A$ and $B$ of width $L_{A}-1$ and $L_{B}-1$ separated by a $(d-1)$-dimensional hyperplane and use the parametrization $x=\left(\boldsymbol{x}_{\|}, x_{\perp}\right)$. To be consistent we fix the boundary conditions to be periodic along $\boldsymbol{x}_{\|}$and open along $x_{\perp}$. For simplicity we fix the size of all the parallel directions to $L$. The boundary term has the form: $H_{\partial}=V+V^{\dagger}$ where $V:=$ $\sum_{\boldsymbol{x}_{\|} \in \partial} c_{\boldsymbol{x}_{\|}, x_{\perp}^{0}}^{A \dagger} c_{\boldsymbol{x}_{\|}, x_{\perp}^{0}}^{B}$ and we can set $x_{\perp}^{0}=1$ without loss of generality. The boundary operator $V$ can then be expressed in terms of the Fourier basis which diagonalize $H_{A / B}$ and turns out to be $V=\sqrt{4 /\left(L_{A} L_{B}\right)} \sum_{\boldsymbol{k}_{\|}, k_{\perp}, q_{\perp}} \sin \left(k_{\perp}\right) \sin \left(q_{\perp}\right) c_{\boldsymbol{k}_{\|}, q_{\perp}}^{A \dagger} c_{\boldsymbol{k}_{\|}, k_{\perp}}^{B}$. Given the form of the one particle dispersion $\epsilon_{k}=2 \sum_{i=1}^{d} \cos \left(k_{i}\right)$ the energy denominator in 
eq. (2) depends only on the perpendicular variables. The entanglement susceptibility takes the form

$$
\chi_{E}=\frac{8}{L_{A} L_{B}} \sum_{\boldsymbol{k}_{\|}, k_{\perp}, q_{\perp}} \frac{\sin ^{2}\left(k_{\perp}\right) \sin ^{2}\left(q_{\perp}\right) n_{k}\left(1-n_{q}\right)}{\left(\epsilon_{k}-\epsilon_{q}\right)^{2}} .
$$

where the occupation numbers are given by $n_{k}=\vartheta\left(-\epsilon_{\boldsymbol{k}_{\|, k_{\perp}}}\right)(\vartheta$ is the Heaviside step function) and we used the notation $q=\left(\boldsymbol{k}_{\|}, q_{\perp}\right)$. For large $L$ we can introduce the function $\Xi\left(k_{\perp}, q_{\perp}\right)=\int d \boldsymbol{k}_{\|} n_{\boldsymbol{k}_{\|}, k_{\perp}}\left(1-n_{\boldsymbol{k}_{\|}, q_{\perp}}\right)$ analogous to $\Xi(q)$ defined in [32]. $\Xi$ measures the volume of $\boldsymbol{k}_{\|}$such that $\left(\boldsymbol{k}_{\|}, k_{\perp}\right)$ lies in the Fermi sea while $\left(\boldsymbol{k}_{\|}, q_{\perp}\right)$ does not. For the specific tight-binding model at half filling $\Xi$ can be computed exactly in terms of a difference of volumes of the standard simplex. In general, for small $\eta:=q-k$, one has $\Xi(k, k+\eta) \propto \eta \vartheta\left(\eta-\sigma_{k}-\sigma_{q}\right)$ where $\sigma_{q / k}=\pi / L_{A / B}$ are the infrared cutoffs in

momentum space. For large sizes one obtains $\chi_{E}=L^{d-1} \int_{\sigma_{k}}^{\pi} d k \int_{\sigma_{k}+\sigma_{q}}^{\pi-k} d \eta f(k, k+\eta)$. Expanding $f(k, k+\eta)=f_{-1}(k) / \eta+\sum_{n=0}^{\infty} f_{n}(k) \eta^{n}$ one realizes that the only divergent contribution is given by the integration of $f_{-1}(k) / \eta$, while all other terms are convergent as $\sigma_{k / q} \rightarrow 0$. The integration over $\eta$ gives trivially a log, and the diverging term turns out to be $\chi_{E} \sim-\alpha_{d} L^{d-1} \ln \left(\sigma_{k}+\sigma_{q}\right)$ where the constant is given by $\alpha_{d}=\int_{0}^{\pi} f_{-1}(k) d k$ 31]. If both $L_{A}$ and $L_{B}$ scale with $L$ we obtain, for large $L$,

$$
\chi_{E} \sim L^{d-1} \ln L .
$$

This result is consistent with the logarithmic violations to the area law for fermionic systems discussed in 32 and 33 . We would like to stress here the two essential ingredients needed to obtain Eq. (8) : i) linearity of $\Xi(k, k+\eta)$ for small $\eta$, more precisely $\Xi(k, k+\eta) \propto \eta \vartheta\left(\eta-\sigma_{k}-\sigma_{q}\right)$; and ii) singularity of the form $(q-k)^{-2}$ for the energy denominators in Eq. (7). These features are believed to be general for free systems and rely on the existence of the Fermi surface and the linearity of the particle-hole excitations close to the Fermi energy. Similar assumptions have been used also in [32. We thus expect Eq. (77) to be valid in more general cases with more complicated geometry and band structures, at least when the above assumptions are satisfied.

\section{Beyond the perturbative regime}

Low energy scaling behavior of a physical quantity is a property of the universality class (in the renormalization group sense) of the Hamiltonian. As long as the perturbation is not strong enough to induce a quantum phase transition scaling behavior is not expected to change. In other words, even though our argument for the entanglement area law relies on a perturbative expansion we expect it to hold true beyond the deep perturbative regime i.e., all the way up to $\lambda=1$ in Eq (11). To elaborate further on this point let us for the moment come back to consider the ground state fidelity. The quantity $\log \mathcal{F}$ between the two ground states of the regions $A$ and $B$ when the boundary coupling $H_{\partial}$ is switched on and off has been considered in [22] and named bi-partite logarithmic fidelity. The authors of [22] provide a heuristic argument, based on the standard quantum mechanics- classical statistical mechanics correspondence, to show that the bi-partite logarithmic fidelity fulfills an area law. The results discussed in this paper so far are tantamount to a rigorous proof of that claim to the second order in $\lambda$. 
To investigate higher order contributions let us notice that for a gapped system with non-degenerate ground state one has $\mathcal{F}(\lambda)=\lim _{\beta \rightarrow \infty} N(\beta) / N^{1 / 2}(2 \beta), N(\beta):=$ $\left\langle e^{-\beta H(\lambda)}\right\rangle$ where $\langle\cdots\rangle$ denotes the quantum-mechanical average over $\left|\Psi_{0}(0)\right\rangle$ [21]. Using the standard interaction picture formula $e^{-\beta H}=e^{-\beta H_{0}} T_{s} \exp \left(-\lambda \int_{0}^{\beta} d s H_{\partial}(s)\right), H_{\partial}(s):=$ $e^{s H_{0}} H_{\partial} e^{-s H_{0}}$ one can prove 24]

$$
\begin{aligned}
& \left\langle T_{s} \exp \left(-\lambda \int_{0}^{\beta} d s H_{\partial}(s)\right)\right\rangle=\exp \left(\sum_{n=1}^{\infty}(-\lambda)^{n} c_{n}(\beta)\right) \\
& c_{n}(\beta)=\int_{0}^{\beta} d s_{1} \cdots \int_{0}^{s_{n-1}} d s_{n}\left\langle H_{\partial}\left(s_{1}\right) \cdots H_{\partial}\left(s_{n}\right)\right\rangle_{c}
\end{aligned}
$$

where the subscript $c$ denotes connected (imaginary time) correlations functions. One can then write:

$$
\log \mathcal{F}(\lambda)=\lim _{\beta \rightarrow \infty} \sum_{n=2}^{\infty}(-\lambda)^{n}\left(c_{n}(\beta)-\frac{1}{2} c_{n}(2 \beta)\right) .
$$

The presence of a spectral gap in $H_{0}$ implies that connected averages scale as $\beta$ or more precisely $c_{n}(\beta)=A_{n} \beta+B_{n}+O\left(e^{-\beta \Delta}\right)\left(A_{n}, B_{n}\right.$ are $\beta$ independent) 24]; whence $\log \mathcal{F}(\lambda)=\frac{1}{2} \sum_{n=2}^{\infty}(-\lambda)^{n} B_{n}$. The term $B_{2}$ is easily seen to yield the fidelity susceptibility $\left(B_{2}=-\chi_{F}\right)$ and therefore, as we have seen, it is upper bounded by $|\partial A|$ in the gapped case.

The key point is now that all these remarks concerning fidelity are directly relevant to Renyi entropy itself. Indeed it turns out that Renyi 2-entropy is a particular instance of logarithmic ground state fidelity 25. To see this fact let us write the purity in the form $\operatorname{Tr} \rho_{A}^{2}=\operatorname{Tr}\left[S_{13}\left|\Psi_{0}(\lambda)\right\rangle\left\langle\left.\Psi_{0}(\lambda)\right|^{\otimes 2}\right]\right.$ where $S_{13}$ is the swap operator between the first and third factor in $\left(\mathcal{H}_{A} \otimes \mathcal{H}_{B}\right)^{\otimes 2}$ i.e., the state space of two copies of the system 23. This trace can be viewed as the ground state fidelity between the ground state $\left|\Psi_{0}(\lambda)\right\rangle^{\otimes 2}$ of $H^{(2)}:=H(\lambda) \otimes \mathbf{1}+\mathbf{1} \otimes H(\lambda)$ and $S_{13}\left|\Psi_{0}(\lambda)\right\rangle^{\otimes 2}$ i.e., the ground state of $S_{13} H^{(2)} S_{13}[25]$. Analog constructions extend to all $\alpha$-entropies with integer $\alpha$ [25]. The fidelity formula (9) shows that one has a direct representation of the Renyi entropy in terms of connected (imaginary time) correlation functions of the boundary perturbation $V_{\partial}:=H_{\partial}^{(2)}-S_{13} H_{\partial}^{(2)} S_{13}$ in a "doubled and twisted" theory. In particular all the entanglement susceptibilities $\chi_{E}^{(n)}:=\partial^{n} S_{2}(0) / \partial \lambda^{n}$ can be expressed by analogous $B_{n}^{\text {twist }}$ terms as defined above, associated with $V_{\partial}$ [26]. Note that, for finite range interactions, $V_{\partial}$ has $O(|\partial A|)$ number of terms and in general represents a surface term. Hence extending to this doubled theory the heuristic statistical mechanics argument of [22] suggests that the area law should indeed hold for all the $\alpha$-Renyi entropies $\left.S_{\alpha}(\rho)=-(\alpha-1)^{-1} \operatorname{Tr} \log \rho^{\alpha}, \alpha \in \mathbf{N}\right)$ in the gapped case with clustering [34]. A deeper analysis of this argument may pave the way towards the rigorous understanding of how area laws for quantum entanglement and their violations arise in physics [35].

\section{Conclusions}

Area laws for quantum entanglement are one of the most common features showing the strong atypicality of physical quantum states in the Hilbert space. In this paper we introduced a natural perturbative object, the entanglement susceptibility (2). Roughly speaking, this quantity measures the rate of entanglement generation (as measured by 
the Renyi 2-entropy) when an infinitesimal boundary interaction term is switched on between two previously decoupled regions. Entanglement susceptibility while much simpler than entanglement entropy itself is able to describe its scaling behavior. This can be seen by bounding entanglement susceptibility with fidelity susceptibility [10, 15. Both area law bounds for gapped systems and possible corrections for critical ones can be then obtained by elementary means. We illustrated the area law bounds for entanglement susceptibility with explicit calculations for the quasi-free systems and logarithmic corrections in the free fermionic critical case.

The non-perturbative regime can also be explored by realizing that Renyi entropy is a particular instance of ground state fidelity in a doubled space 25. This unification allows one to express entanglement by an exact series expansion of integrated connected correlations of a twisted boundary interaction.

Let us conclude by mentioning a couple of natural goals for future research. On the experimental side, very recent work indicates that entanglement susceptibility may be directly measurable in bosonic optical lattices [36. On the theoretical side, we would like to extend the results of this paper to excited energy eigenstates of local Hamiltonians [37: Are they going to be more alike typical quantum states?

Acknowledgments The authors acknowledge partial support by the ARO MURI grant W911NF-11-1-0268. PZ also acknowledges partial support by NSF grants No. PHY-969969 and No. PHY-803304. We would like to thank H. Saleur, S. Haas, A. Hamma and D. Lidar for useful comments.

\section{References}

[1] D. Poulin, A. Qarry, R. D. Somma, F. Verstraete, Phys. Rev. Lett. 106, 170501(2011)

[2] P. Hayden, D. W. Leung, A. Winter, Comm. Math. Phys. 265, 95-117, (2006)

[3] M.M. Wolf, F. Verstraete, M.B. Hastings, J.I. Cirac, Phys. Rev. Lett. 100, 070502 (2008)

[4] J. Eisert, M. Cramer and M. B. Plenio Rev. Mod. Phys. 82, 277 (2010)

[5] L. Amico, R. Fazio, A. Osterloh, V. Vedral, Rev. Mod. Phys.80, 517 (2008)

[6] A. Hamma, R. Ionicioiu, and P. Zanardi, Phys. Lett. A 337, 22 (2005); A. Kitaev and J. Preskill, Phys. Rev. Lett. 96, 110404 (2006); M. Levin and X.-G. Wen, ibid. 110405 (2006).

[7] A. Hamma, S. Santra and P. Zanardi, Phys. Rev. Lett. 109, 040502 (2012); A. Hamma, S. Santra and P. Zanardi, Phys. Rev. A 86, 052324 (2012)

[8] G. Vidal, Phys. Rev. Lett. 91, 147902 (2003)

[9] For example there exist states with finite correlation length but exponentially large entanglement: M. Hastings, Phys. Rev. B 76, 035114 (2007)

[10] P. Zanardi and N. PaunkoviĆ, Phys. Rev. E 74, 031123 (2006); H. Q. Zhou and J. P. Barjaktarevic, J. Phys. A: Math. Theor. 41412001 (2008).

[11] W-L. You, Y-W Li, S-J. Gu, Phys. Rev. E 76, 022101 (2007)

[12] L. Campos Venuti and P. Zanardi, Phys. Rev. Lett. 99, 095701 (2007).

[13] S. Sachdev, Quantum Phase Transitions (Cambridge University Press, (1999)

[14] In the case of a multi parameter boundary coupling $H_{\partial}:=\sum_{\mu} \lambda_{\mu} V_{\mu}$ the entanglement susceptibility can be straightforwardly generalized to a tensor $\left(\chi_{E}\right)_{\mu \nu}=$ $\partial^{2} S_{2}(0) / \partial \lambda_{\mu} \partial \lambda_{\nu}$. This is a degenerate metric tensor over the parameter manifold.

[15] P. Zanardi, P. Giorda and M. Cozzini, Phys. Rev. Lett. 99, 100603 (2007)

[16] With the notation $H_{\partial}^{A / B}(\tau)=e^{\tau H_{A / B}} H_{\partial} e^{-\tau H_{A / B}}$ and $\langle\cdot\rangle_{c}$ denoting connected correlations on the ground state, one can also show that $\chi_{E}=\chi_{F}-$ $\int_{0}^{\infty} d \tau \tau\left[\left\langle H_{\partial}^{A}(\tau) H_{\partial}\right\rangle_{c}+\left\langle H_{\partial}^{B}(\tau) H_{\partial}\right\rangle_{c}\right]$.

[17] M. B. Hastings, Phys. Rev. Lett. 93 140402(2004)

[18] F. Verstraete, M.M. Wolf, D. Perez-Garcia and J.I. Cirac, Phys. Rev. Lett. 96, 220601 (2006)

[19] M. Z. Hasan and C. L. Kane, Rev. Mod. Phys., 82, 3045-3067, (2010)

[20] L. Masanes, Phys. Rev. A 80, 052104 (2009). 
[21] Here we assumed that the overlap of $\left\langle\Psi_{0}(\lambda) \mid \Psi_{0}(0)\right\rangle$ is non vanishing (for finite system size).

[22] J. Dubail, J-M. Stephan, J. Stat. Mech. L03002 (2011)

[23] P. Zanardi, C. Zalka and L. Faoro. Phys. Rev. A 62, 030301 (2000)

[24] R. Kubo, J. Phys. Soc. Jpn. 17, 1100-1120 (1962)

[25] J. Cardy, Phys.Rev.Lett. 106, 150404 (2011)

[26] For example: computing the fidelity susceptibility of the doubled and twisted theory gives an alternative and straightforward way of proving (2).

[27] By this we mean that the, for all $i \in \Lambda$ the sets $\mathcal{V}_{i}=\left\{j \in \Lambda / V_{i j} \neq 0\right\}$ have size $O(1)$.

[28] R. Bhatia, Matrix Analysis, Springer (1997)

[29] S. Garnerone, N. T. Jacobson, S. Haas and P. Zanardi, Phys. Rev. Lett. 102, 057205 (2009)

[30] M. Cramer and J. Eisert, New. J. Phys. 8, 71 (2006)

[31] In the present case $f_{-1}(k)=2 /\left[\pi^{d+1}(d-2) !\right](\pi-k) \sin (k)^{2}$ and $\alpha_{1}=2 / \pi^{2}=0.202$, $\alpha_{2}=1 / \pi^{2}=0.101, \alpha_{3}=1 /\left(2 \pi^{2}\right)=0.05$.

[32] M.M. Wolf, Phys. Rev. Lett. 96, 010404 (2006)

[33] D. Gioev and I. Klich, Phys. Rev. Lett. 96, 100503 (2006)

[34] Notice that also the entanglement susceptibility notion can be easily extended to the general $\alpha$-Renyi entropy $S_{\alpha}$ for $(\alpha \in(1,+\infty))$. In this case one can easily show that $S_{\alpha}\left(\rho_{A}(\lambda)\right)=-\frac{\alpha}{\alpha-1} \lambda^{2} \chi_{E}+O\left(\lambda^{3}\right)$. When $\alpha \rightarrow 1^{+}$the $\alpha$-Renyi entropy converges to the von Neumann entanglement entropy $S(\rho):=-\operatorname{tr} \rho \log \rho$ but $d^{2} S_{\alpha}(0) / d \lambda^{2}=$ $-\alpha(\alpha-1)^{-1} \chi_{E}$ diverges to $-\infty$. The susceptibility is not defined for the von Neumann as this latter is not differentiable at pure states.

[35] One should prove that all the $B_{n}^{t w i s t}$ 's are $O(|\partial A|)$ and that the series $\frac{1}{2} \sum_{n=2}^{\infty}(-\lambda)^{n} B_{n}^{t w i s t}=S_{2}(\lambda)$ has a finite convergence radius.

[36] A. J. Daley, H. Pichler, J. Schachenmayer, P. Zoller, Phys. Rev. Lett. 109, 020505 (2012)

[37] V. Alba, M. Fagotti and P. Calabrese, J. Stat. Mech., P10020 (2009)

\section{Appendix}

Proof of (2). Let us expand the ground state of (1) in powers of $\lambda$ : $\left|\Psi_{0}(\lambda)\right\rangle=\mathcal{N} \sum_{n=0}^{\infty} \lambda^{n}\left|\Psi^{(n)}\right\rangle\left(\left|\Psi^{(0)}\right\rangle=\left|0_{A}\right\rangle\left|0_{B}\right\rangle.\right)$ The perturbative eigenvector corrections $\left|\Psi^{(n)}\right\rangle$ are given by the elementary perturbation theory: $\left|\Psi^{(n)}\right\rangle=$ $G_{0}\left(E_{0}\right)\left(H_{\partial}\left|\Psi^{(n-1)}\right\rangle-\sum_{k=0}^{n-1} E^{(n-k)}\left|\Psi^{(k)}\right\rangle\right)$; where $E^{(k)}=$ k-th order correction to the unperturbed eigenenergy $E^{(0)}=E_{0}, G_{0}:=\sum_{n>0}\left(E_{0}-E_{n}\right)^{-1}\left|\Psi_{n}\right\rangle\left\langle\Psi_{n}\right|=Q\left(E_{0}-\right.$ $\left.H_{0}\right)^{-1} Q$ is the (projected) resolvent of $H_{0}\left(Q:=1-\left|\Psi_{0}\right\rangle\left\langle\Psi_{0}\right|\right.$.) The reduced density matrix is given by $\rho_{A}(\lambda)=\mathcal{N}^{2} \sum_{n=0}^{\infty} \lambda^{n} \rho^{(n)}$ where $\rho^{(n)}:=\sum_{k=0}^{n} \operatorname{Tr}_{B}\left|\Psi^{(n-k)}\right\rangle\left\langle\Psi^{(k)}\right|$. Let us now consider the leading contributions $n=0,1$; performing the partial trace over the region $B$ one finds

$$
\begin{aligned}
& \rho^{(0)}=\left|0_{A}\right\rangle\left\langle 0_{A}\left|, \quad \rho^{(1)}=\sum_{p_{A}>0} C\left(p_{A}, 0\right)\right| 0_{A}\right\rangle\left\langle p_{A}\right|+\text { h.c, } \\
& \rho^{(2)}=\sum_{p_{A}, q_{A}}\left|p_{A}\right\rangle\left\langle q_{A}\right| \sum_{p_{B}} C\left(p_{A}, p_{B}\right) \overline{C\left(q_{A}, p_{B}\right)} .
\end{aligned}
$$

in which $C\left(p_{A}, p_{B}\right)=\left\langle p_{A} p_{B}\left|H_{\partial}\right| 0_{A} 0_{B}\right\rangle /\left(E_{p_{A}}+E_{p_{B}}-E_{0}\right)$. Let us now compute the purity of $\rho_{A}$ : $\operatorname{Tr} \rho_{A}^{2}=\mathcal{N}^{4} \operatorname{Tr}\left(\left(\rho^{(0)}\right)^{2}+\lambda^{2}\left(\rho^{(1)}\right)^{2}+\lambda^{2}\left\{\rho^{(0)}, \rho^{(2)}\right\}\right)+O\left(\lambda^{3}\right)$. Here one has to notice that $\operatorname{Tr}\left\{\rho^{(0)}, \rho^{(1)}\right\}=0, \operatorname{Tr}\left\{\rho^{(0)}, \rho^{(2)}\right\}=2 \sum_{p_{B}>0}\left|C\left(0, p_{B}\right)\right|^{2}$, and $\operatorname{Tr}\left(\rho^{(1)}\right)^{2}=2 \sum_{p_{A}>0}\left|C\left(p_{A}, 0\right)\right|^{2}$. The normalization factor at this order is given by $\mathcal{N}^{-2}=\|\left|\Psi_{0}\right\rangle+\left|\Psi^{(1)}\right\rangle \|^{2}=1+\lambda^{2} \sum_{p_{A}+p_{B}>0}\left|C\left(p_{A}, p_{B}\right)\right|^{2}$. Using this explicit form and expanding again at leading order one obtains $\operatorname{Tr} \rho_{A}^{2}=1-2 \lambda^{2} \sum_{p_{A}, p_{B} \geq 1}\left|C\left(p_{A}, p_{B}\right)\right|^{2}+$ $O\left(\lambda^{3}\right)$. Finally taking the negative of $\log$ one finds, the expression (2).

This derivation shows that the appearance of the fidelity susceptibility in our entanglement argument while remarkable is certainly not surprising. Indeed 
an important ingredient in the derivation above is the the normalization factor $1 / \mathcal{N}^{2}=\| \sum_{n=0}^{\infty} \lambda^{n}\left|\Psi^{(n)}\right\rangle \|^{2}$. Since in standard Rayleigh-Schrödinger perturbation theory one can always choose $\left\langle\Psi^{(0)} \mid \Psi^{(k)}\right\rangle=0$ for $k>0$, it follows that $\mathcal{N}^{2}$ is nothing but the ground state fidelity: $\mathcal{F}^{2}=\left|\left\langle\Psi_{0}(0) \mid \Psi_{0}(\lambda)\right\rangle\right|^{2}=\left|\left\langle\Psi^{(0)} \mid \Psi_{0}(\lambda)\right\rangle\right|^{2}=$ $\mathcal{N}^{2}\left|\sum_{n=0}^{\infty}\left\langle\Psi_{0} \mid \Psi^{(k)}\right\rangle\right|^{2}=\mathcal{N}^{2}$. 